\title{
Recursos hídricos actuales y futuros en Chile y su disponibilidad para la vegetación mediterránea
}

\section{Current and future water resources in Chile and its availability for the Mediterranean vegetation}

\author{
Javier Lozano-Parra, Instituto de Geografía, Pontificia Universidad Católica de Chile, Chile, \\ jlozano@outlook.es \\ (1) https://orcid.org/0000-0002-7890-9148
}

Ramón García-Marín, Departamento de Geografía, Universidad de Murcia, España, ramongm@um.es

(1) https://orcid.org/0000-0003-3268-5182

Manuel Pulido, Grupo de Investigación GeoAmbiental, Universidad de Extremadura, España, mapulidof@unex.es

(1) https://orcid.org/0000-0001-9340-0107

Víctor Ruiz-Álvarez, Departamento de Geografía, Universidad de Murcia, España, victor.ruiz1@um.es (1) https://orcid.org/0000-0002-6630-4571

Resumen: Las regiones áridas y semiáridas cubren actualmente más del $45 \%$ de la superficie terrestre. El territorio central de Chile se encuentra entre estas zonas y constituye un espacio que debe ser conservado por su importancia económica, ecológica y climática. Sin embargo, la creciente presión sobre el agua y las modificaciones climáticas hacen necesario cuantificar la cantidad de recursos hídricos, tanto actuales como futuros, distribuidos por el territorio chileno y establecer qué biomas podrían verse más afectados por las variaciones hídricas. Este estudio cuantifica el balance hídrico actual y futuro de forma distribuida para todo el territorio chileno y establece qué formaciones vegetales mediterráneas serán las más afectadas por las variaciones de los recursos hídricos. Los resultados demuestran que la zona central, lugar donde se concentra la mayor parte de la población chilena, constituiría uno de los territorios más sensibles a la escasez hídrica, con reducciones de los recursos que alcanzan un promedio de $75 \mathrm{~mm}$ año-1 para el $80 \%$ de los espacios mediterráneos. El descenso de los recursos hídricos podría comprometer el funcionamiento ambiental y económico de los espacios mediterráneos de Chile.

Palabras clave: Balance hídrico; Cambio climático; Áreas frágiles; Clima mediterráneo; Chile.

Abstract: The arid and semi-arid regions occupy more than $45 \%$ of global land surface, including the central territory of Chile. The latter represents a region which should be preserved due to its economic, ecological and climatic values. Nonetheless, the increasing pressure on water and climate change suggest the quantification of its present and future water resources as well as to know what biomes could be affected by these hydrological variations. So, we have quantified the current and future water balances for whole Chile and investigated about the Mediterranean vegetation formations more affected by the variations in the water amount. The results have shown that the central part of the country, where inhabits the most of Chilean population, is the territory more sensitive to water scarcity since reductions in water can represent an average of $75 \mathrm{~mm} \mathrm{y}^{-1}$ for $80 \%$ of the Mediterranean biome. This reduction could suppose these areas do not work properly any more.

Keywords: Water balance; Climate change; Sensitive areas; Mediterranean climate type; Chile. 


\section{Introducción}

Chile es uno de los países con mayores recursos hídricos por persona y de los que ostenta mayor superficie y diversidad de biomas del mundo (World Bank, 2011). Sin embargo, debido a la presión creciente sobre los recursos hídricos se hace necesario afrontar las cuestiones relacionadas con el agua desde enfoques que permitan solventar los problemas y gestionar eficientemente los recursos. Uno de estos enfoques es el ecohidrológico, es decir, aquel que aborda desde una perspectiva combinada los procesos hidrológicos y ecológicos o, dicho de otro modo, aquel que aborda las interacciones entre el agua y la vegetación. Una tarea primordial para la ecohidrología es entender las respuestas surgidas en los diferentes ecosistemas ante un medioambiente cambiante, ya que ello permitirá mejorar la gestión de los espacios y predecir sus dinámicas (García Marín et al., 2010; Lozano-Parra, 2015; RodríguezIturbe, 2000; Rodríguez-Iturbe et al., 2007).

La ecohidrología se establece como disciplina científica independiente a principios de este siglo (Eagleson, 2002) y se centra en el estudio de las relaciones agua-planta. A comienzos de siglo, la UNESCO desarrolló un programa específico para estudiar los procesos ecohidrológicos a nivel mundial, a fin de entender las interacciones entre agua y plantas a escala global. A pesar de ello, los estudios científicos desarrollados bajo este enfoque son todavía muy escasos en Chile, de modo que la ecohidrología chilena del presente siglo se enfrenta a importantes desafíos, como el aumento de la presión sobre el agua, la disminución de los recursos o el cambio climático, aspectos que has sido destacados por investigadores como Blöschl et al. (2019) o Asbjornsen et al. (2011).

Los estudios ecohidrológicos han puesto de manifiesto que la presencia de vegetación es causa y consecuencia de la presencia de agua, y viceversa (Rodríguez-lturbe y Porporato, 2004). Por ejemplo, la distribución espacial de la vegetación ejerce un fuerte control sobre la variabilidad espacio-temporal de la contenidos hídricos del suelo y sobre procesos como la infiltración o la redistribución del agua (Lozano-Parra et al., 2015; 2016), mientras que la disponibilidad de agua en el suelo determina el crecimiento y desarrollo de la vegetación y su productividad (Lozano-Parra y Schnabel, 2015; Lozano-Parra et al., 2014).

Los procesos ecohidrológicos son cada vez mejor comprendidos debido a la utilización de métodos y herramientas avanzadas, como los modelos o los sensores especializados. Sin embargo, los procesos de retroalimentación a diferentes escalas espacio-temporales aun no son bien entendidos debido a que las relaciones multiescalares originadas entre los procesos ecohidrológicos son no-lineales (Maneta et al., 2018). Ello desemboca en la existencia de un comportamiento no-unidireccional para una misma variable, es decir, que dicha variable puede presentar diferentes estados estables cuyos comportamientos pueden basarse en la superación de ciertos umbrales (Scheffer y Carpenter, 2003; Scheffer et al., 2001). Esto significaría que, por ejemplo, zonas que han sido húmedas y que actualmente están padeciendo importantes variaciones en sus condiciones hídricas podrían modificar sus 
características ambientales hacia estados más secos en los que persistirian prolongadamente de forma estable.

El estudio de los procesos ecohidrológicos a diferentes escalas se torna, por tanto, necesario para entender la dinámica de los ecosistemas y las variaciones que pueden tener los recursos naturales. El análisis en amplias superficies puede ayudar a identificar los mecanismos que inducen a la heterogeneidad espacial, los cuales son particularmente importantes en ecosistemas donde el estrés hídrico limita el crecimiento de las plantas, como los que se encuentran en la zona central y norte de Chile (Asbjornsen et al., 2011; Rodríguez-Iturbe y Porporato, 2004). Dichas zonas, al ser las más pobladas, se muestran muy sensibles a la variación de los recursos hídricos (McColl et al., 2017).

Debido a la importancia que tienen los recursos hídricos para el mantenimiento y desarrollo de la vegetación, este trabajo pretende definir la cantidad de recursos hídricos que ostenta Chile en la actualidad y que ostentará en el futuro, y establecer qué biomas podrían verse más afectados por la variación de dichos recursos. De esta forma, los objetivos de este estudio son los siguientes: I) determinar el balance hídrico actual de forma distribuida para todo el territorio de Chile; II) definir el balance hídrico futuro para Chile en función de diferentes escenarios climáticos y III) determinar qué biomas de la zona mediterránea de Chile serán los más afectados por las futuras variaciones de los recursos hídricos.

\section{2. Área de estudio}

Chile abarca una superficie sensiblemente superior a los 2 millones de $\mathrm{km}^{2}$ y alcanza una longitud superior a los 8000 km, desde el límite con Perú hasta el Polo Antártico. Tiene una población próxima a los 18 millones de habitantes concentrada principalmente en la zona central del país.

Su geología está determinada fundamentalmente por su situación al borde occidental de la placa tectónica sudamericana, lo que le ha permitido interactuar con las placas subducentes ubicadas hacia el oeste, resultando en unos rasgos geológicos muy característicos. Debido a esto, su geomorfología puede considerarse extraordinariamente variada y compleja. Las formas actuales tienen su origen en los procesos de erosión de material las zonas elevadas y su posterior acumulación y sedimentación en cuencas y depresiones. Asimismo, tanto el glaciarismo como la acción volcánica o la acción fluvial han generado relieves presentes a lo largo de todo el país. Los relieves predominantes son la Cordillera de los Andes, Cordillera de la Costa, Depresión Intermedia y los Valles Transversales.

El clima está determinado por el gradiente latitudinal y altitudinal, y por la influencia marítima del Océano Pacífico. Debido a ello, las variaciones climáticas más importantes se producen por efectos de la latitud y de la elevación, abarcando casi todos los dominios climáticos a excepción de algunos pocos, como por ejemplo el tropical Iluvioso (Figura 1). 
Los suelos son muy variados como causa de sus diferentes factores genéticos y evolutivos, que vienen determinados principalmente por el relieve, extensión latitudinal del territorio, continentalidad y variedad climática. Los principales tipos de suelos son los Cambisoles, Andosoles, Regosoles y Leptosoles (Figura 1).

La vegetación chilena, al igual que el clima, está condicionada por el gradiente latitudinal y altitudinal. Como consecuencia, presenta una gran diversidad de recursos bióticos caracterizados por un fuerte endemismo (se estima que existen aproximadamente más de 6.300 especies endémicas). A pesar de ello, la diversidad vegetal es menor que la encontrada en países vecinos como Brasil.
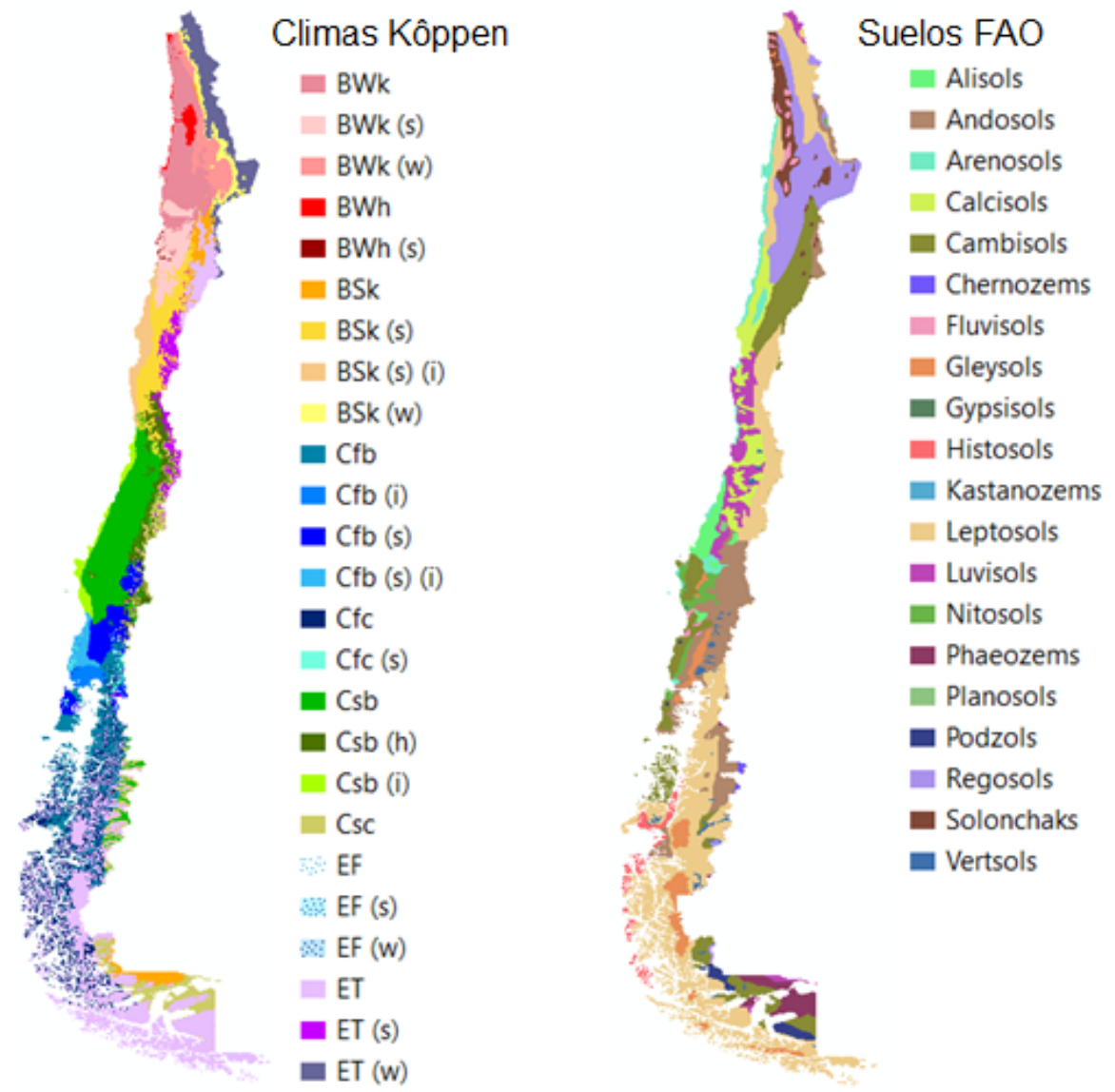

Figura 1: Climas y suelos de Chile, según Rubel y Kottek (2010) y FAO (2014).

\section{Metodología}

3.1. Obtención de variables climáticas espacialmente distribuidas actuales y futuras

Las variables climáticas distribuidas de forma continua en el espacio fueron obtenidas a partir de Fick y Hijmans (2017). Para ello, se partió de valores puntuales de precipitación mensual, temperatura media mensual y temperaturas mínima y máxima $y$, posteriormente, se procedió a la interpolación de dichas variables con el fin de generar una malla espacialmente continua a escala mundial con una resolución de 1 
$\mathrm{km}^{2}$. El método de interpolación está basado en splines suavizados que operan considerando análisis de covarianza y distribuciones espaciales del error estándar. Dicho método puede ser visto como una generalización de la regresión multivariable estándar, en la cual el modelo paramétrico es reemplazado por una función noparamétrica suavizada (Wahba, 1990). Este algoritmo de interpolación emplea variables climáticas, latitud, longitud y elevación como componentes independientes de entrada. Esta última variable fue obtenida desde la Shuttle Radar Topography Mission (SRTM) de la NASA. Las series climáticas puntuales fueron obtenidas a partir de estaciones meteorológicas distribuidas globalmente y pertenecientes a la Global Historical Climatology Network (GHCN). Con el fin de aumentar el número de estaciones y mejorar las series de datos, también fueron utilizadas estaciones meteorológicas procedentes de otras fuentes, como la Food and Agriculture Organization (FAO), la World Meteorological Organization (WMO), el International Center for Tropical Agriculture (CIAT) o la R-HydroNET.

Con el propósito de determinar el balance hídrico actual de forma distribuida y de estimar el impacto que tendrán las variaciones futuras sobre los recursos hídricos y los principales biomas de Chile, se utilizaron dos periodos temporales. El primero hace referencia al periodo 1970-2000 (periodo base); mientras que el segundo hace referencia a un escenario representativo de concentración de gases de efecto invernadero para el año 2050, el RCP4.5, que asume un descenso en la concentración de gases a partir del año 2050. Las proyecciones utilizadas fueron generadas por MIROC5, uno de los modelos climáticos globales (GCMs) que forman parte de la quinta fase del proyecto de inter-comparación de modelos acoplados (CMIP5).

\subsection{Cálculo del balance hídrico y obtención de las formaciones vegetales}

La distribución espacialmente continua de los recursos hídricos en Chile fue obtenida mediante el cálculo de la reserva de agua. Para ello se determinó el balance hídrico a escala de píxel, expresado como sigue:

$$
\beta=P-E
$$

Donde $\beta$ es la reserva de agua o disponibilidad de recursos hídricos $(\mathrm{mm}), \mathrm{P}$ son las entradas de agua por precipitación $(\mathrm{mm})$ y $\mathrm{E}$ son las salidas de agua por evapotranspiración $(\mathrm{mm})$.

La evapotranspiración fue calculada mediante el método de Turc [1961], que permite obtener la evapotranspiración real anual a partir de la siguiente expresión:

$$
E=\frac{P}{\sqrt{C+\frac{P^{2}}{L^{2}}}}
$$


Donde $E$ es la evapotranspiración real anual (mm año-1), $P$ es la precipitación anual $(\mathrm{mm}$ año-1) y $\mathrm{C}$ es un valor constante. Por último, $\mathrm{L}$ es un coeficiente que está en función de la temperatura $(\mathrm{T})$ media anual $\left({ }^{\circ} \mathrm{C}\right)$ y se obtiene de la siguiente forma:

$$
L=300+25 T+0.05 T^{3}
$$

La distribución espacial de los principales biomas de Chile fue obtenida a partir de la clasificación desarrollada por Pliscoff y Luebert (2006). Dicha clasificación propone la existencia de cuatro macrobioclimas a lo largo del territorio y de 125 unidades básicas denominadas pisos de vegetación. Cada piso constituye un espacio caracterizado por un conjunto de comunidades vegetales con estructura y fisionomía uniforme, situadas bajo condiciones mesoclimáticamente homogéneas que ocupan una posición determinada a lo largo de un gradiente altitudinal y a una escala espacio-temporal específica. Posteriormente, un piso de vegetación puede ser caracterizado por su composición florística, su dinámica y su heterogeneidad interna (Pliscoff y Luebert, 2006).

\section{Resultados y discusión}

\subsection{Recursos hídricos actuales y futuros en Chile}

Las entradas de agua por precipitación a lo largo de todo el territorio chileno alcanzaron un promedio de 1006 mm (Figura 2.A) durante el periodo 1970-2000, lo que supuso un volumen anual de $761.5 \mathrm{~km}^{3}$ de agua. De esta cantidad, un promedio de $311 \mathrm{~mm}$ fue devuelto a la atmósfera mediante el proceso de evapotranspiración (Figura 2.B), lo que constituyó un volumen anual de agua de aproximadamente 235 $\mathrm{km}^{3}$. De esta forma, los excedentes generados por el territorio supusieron un promedio hídrico de $669 \mathrm{~mm}$ anuales (Figura 2.C), una cantidad equivalente a un volumen que supera los $500 \mathrm{~km}^{3}$ de agua, los cuales son susceptibles de ser utilizados por el hombre cada año. A pesar de esta abundancia de agua, la disponibilidad de recursos es variable en el espacio y en el tiempo. Las regiones del norte de Chile presentaron unos excedentes hídricos muy bajos. Esto contrasta con las regiones del sur, más ricas en recursos hídricos (Figura 2.C). La zona central registró la mayor variabilidad espacial, donde algunos espacios mostraron unos excedentes hídricos exiguos y otros una mayor abundancia. 

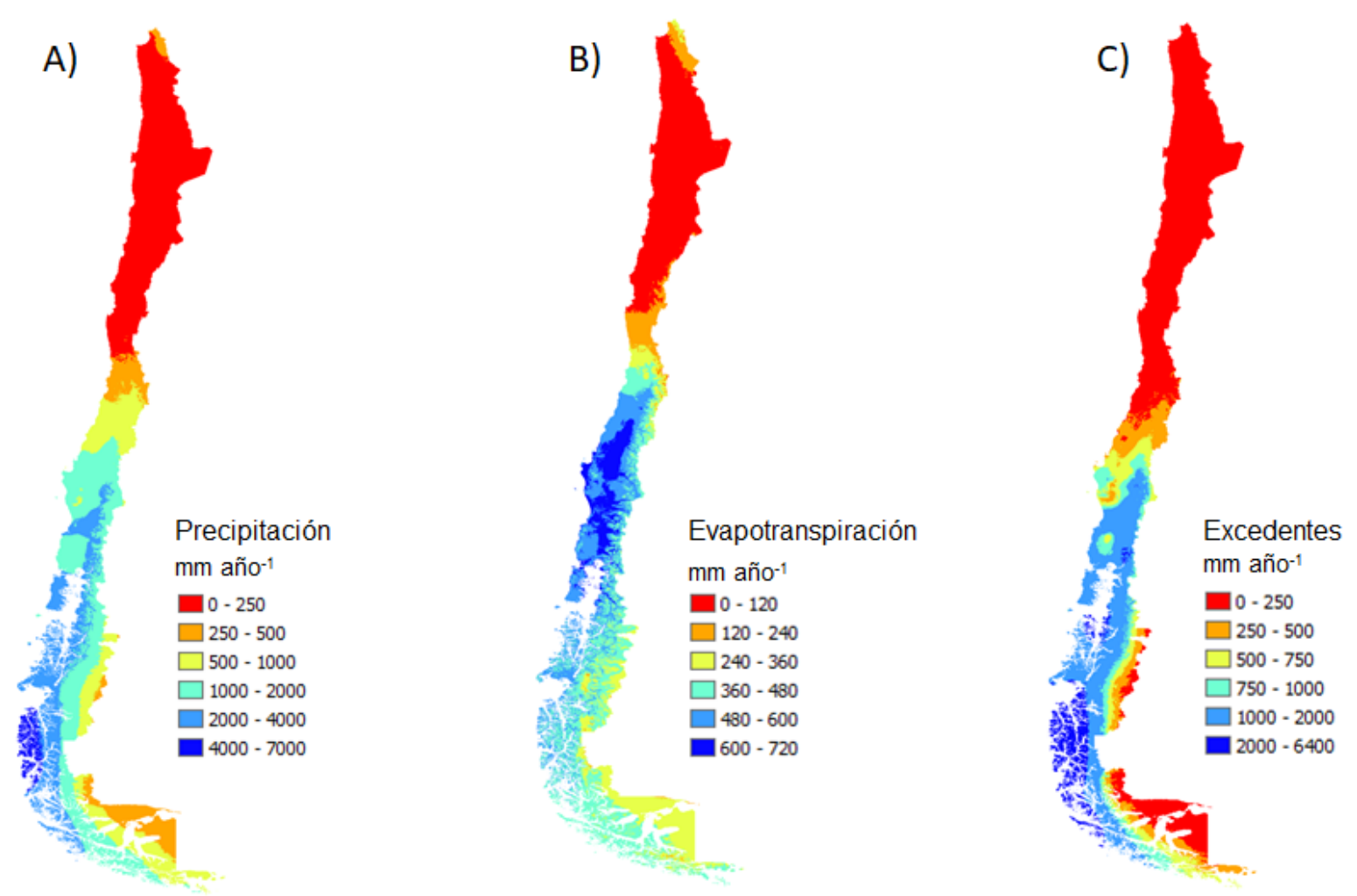

Figura 2: Principales procesos del ciclo hidrológico (Precipitaciones, A; Evapotranspiración, B; y Excedentes de agua, C) en Chile durante el periodo base de 30 años (1970-2000).

La disponibilidad varió cuando los recursos fueron considerados por regiones administrativas en función de su población. La abundante disponibilidad hídrica del sur, escasamente poblado, contrastó con la insuficiencia de recursos en zonas con mayores densidades de población, como el centro y el norte (Figura 3). Las regiones más sensibles se localizarían en la zona central de Chile, donde la densidad de población es mayor, la actividad empresarial más intensa, y la abundancia de recursos hídricos no es tan elevada como en las zonas húmedas del sur. De esta forma, la Región Metropolitana se establece como el territorio con menos recursos hídricos $\left(\mathrm{m}^{3}\right.$ por habitante y año) de Chile después de las regiones de Antofagasta y Tarapacá. Opuestamente, las regiones con mayores recursos fueron, por este orden, Aysén, Magallanes y Los Lagos. 


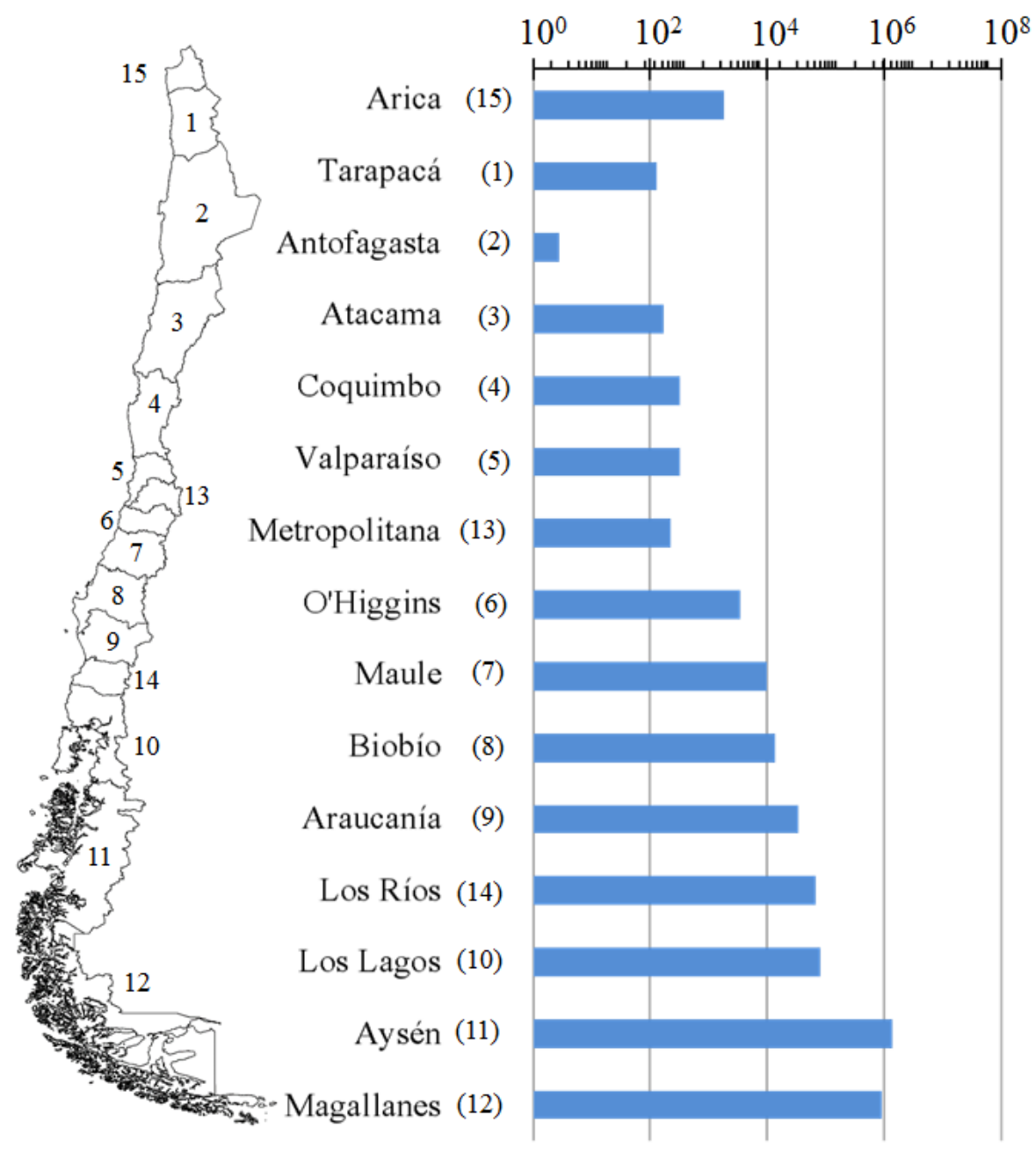

Figura 3: Disponibilidad hídrica por persona ( $\mathrm{m}^{3}$ habitante/año) según las distintas regiones de Chile para el periodo $1970-2000$.

A pesar de la riqueza en recursos hídricos que muestra Chile, según los últimos pronósticos del IPCC (2014), dichos recursos podrían ser cada vez más escasos debido a las modificaciones en los procesos hidrometeorológicos provocados por el cambio climático. Las proyecciones realizadas para el año 2050 por MIROC5 para el escenario RCP4.5 muestran que las entradas de agua por precipitación alcanzarán un promedio de $938 \mathrm{~mm}_{\text {año-1 }}$ (Figura 4.A), lo que supone una reducción del $7 \%$ en las precipitaciones con respecto al periodo base. Esta cantidad equivale a una disminución media superior a los $50 \mathrm{~km}^{3}$ de agua. Del mismo modo, la evapotranspiración real media alcanzará valores un $3 \%$ mayores que los del periodo

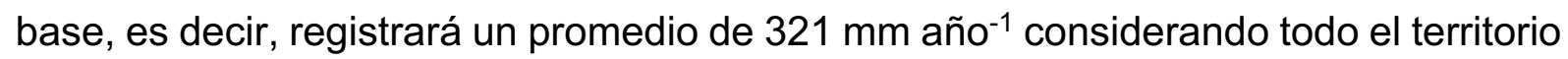
chileno (Figura 4.B), lo que equivale a un aumento medio de $8 \mathrm{~km}^{3}$ de agua que serán devueltos a la atmósfera. Por último, los excedentes generados por el territorio chileno

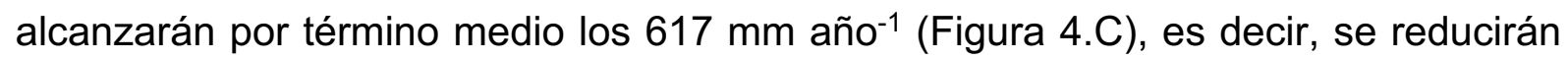
un $8 \%$ con respecto al periodo base, lo que supone una disminución de aproximadamente $40 \mathrm{~km}^{3}$ de los recursos hídricos susceptibles de ser utilizados por el hombre cada año. 

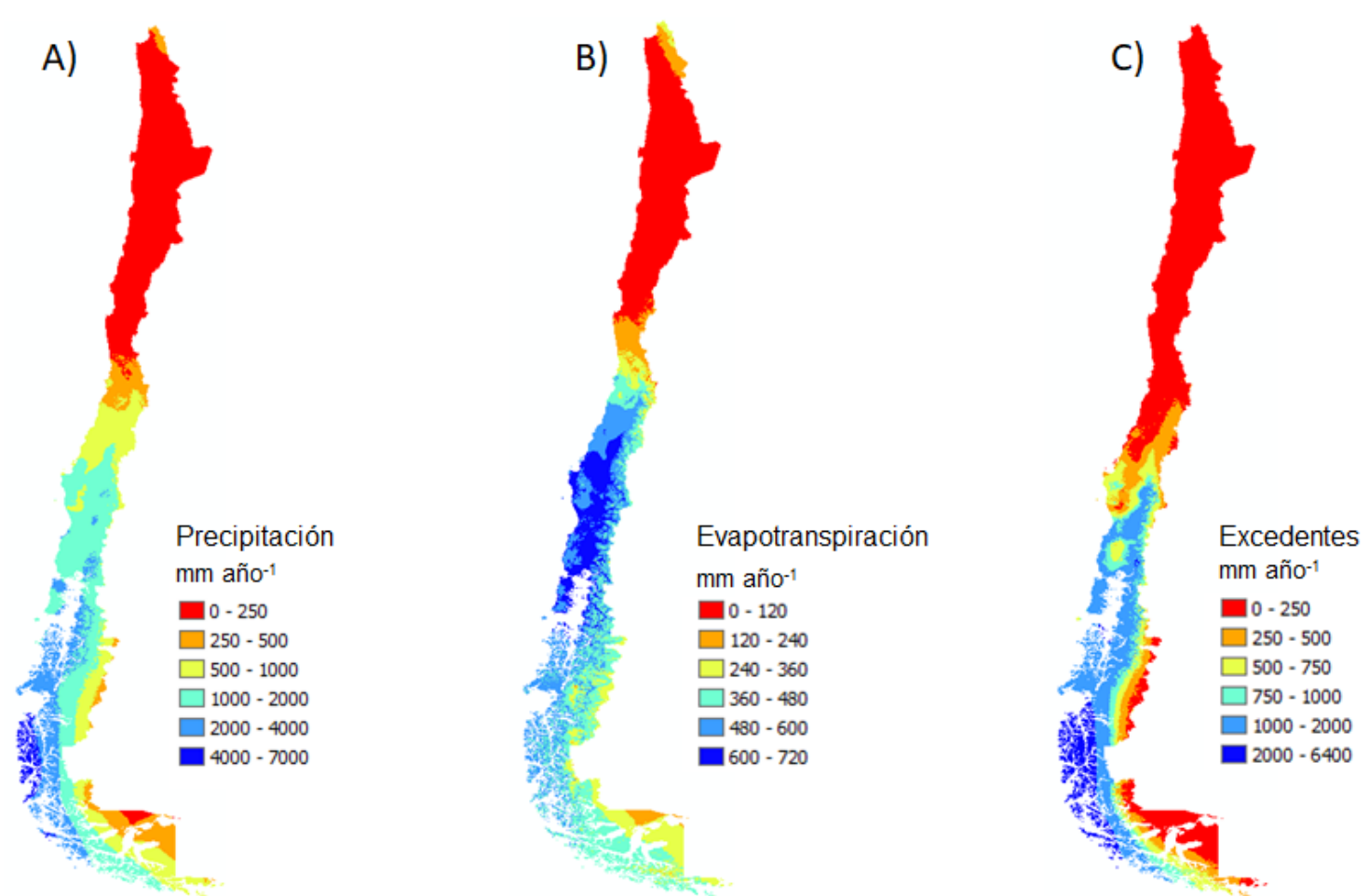

Figura 4: Principales procesos del ciclo hidrológico (Precipitaciones, A; Evapotranspiración, B; y Excedentes de agua, C) en Chile para el escenario RCP4.5 del año 2050.

Pese a los posibles efectos del cambio climático, la demanda hídrica continúa aumentando. Por ejemplo, la DGA continua cerrando nuevos derechos de agua para acuíferos en el norte de Chile, ya que la extracción está próxima a sobrepasar los umbrales de recarga natural de los acuíferos (Hearne y Donoso, 2014). En el norte de Chile, la demanda hídrica por las empresas mineras está relacionada con el aumento en los precios del cobre (Rivera et al., 2016). Sectores como el energético, también intensifican la presión sobre los recursos hídricos ya que las compañías hidroeléctricas sostienen una demanda creciente para abastecer la expansión industrial (Larraín y Poo, 2010). La demanda de la agricultura también está creciendo a medida que la población aumenta y que los productos compiten en los mercados globales (Rodell et al., 2018). De este modo, tanto la escasez como la competencia por los recursos hídricos aumentan a lo largo de todo el país y la disparidad espacial en el reparto de los recursos se ve reflejada en el aumento de los conflictos por el agua (Larraín y Poo, 2010). La concentración de disputas se localiza en la zona central y norte del país donde los recursos hídricos son más variables y la demanda es más intensa (Rivera et al., 2016).

4.2. Variación de los recursos hídricos y disponibilidad para las formaciones vegetales mediterráneas

Las proyecciones realizadas para el escenario RCP4.5 del año 2050 por MIROC5 muestran que el impacto del cambio climático sobre los recursos hídricos de Chile 
podría ser mayor en la zona central y centro-sur, donde se ubica buena parte de la vegetación mediterránea. Las proyecciones revelan que casi todo el territorio chileno podría padecer un descenso de sus recursos hídricos, es decir, que experimentaría una reducción en la cantidad de agua que resulta de la diferencia entre las precipitaciones y la evapotranspiración (Figura 5.A). Únicamente zonas del sur de Chile y espacios muy concretos de la zona central, correspondientes a zonas andinas con elevada altitud, experimentarían aumentos en sus reservas hídricas. El mayor descenso de los recursos hídricos se producirá en territorios del centro-sur del país, con una reducción que podría alcanzar los $500 \mathrm{~mm}$ año-1 menos con respecto al periodo base, llegando incluso a ser inferiores en zonas de la región de Los Lagos.
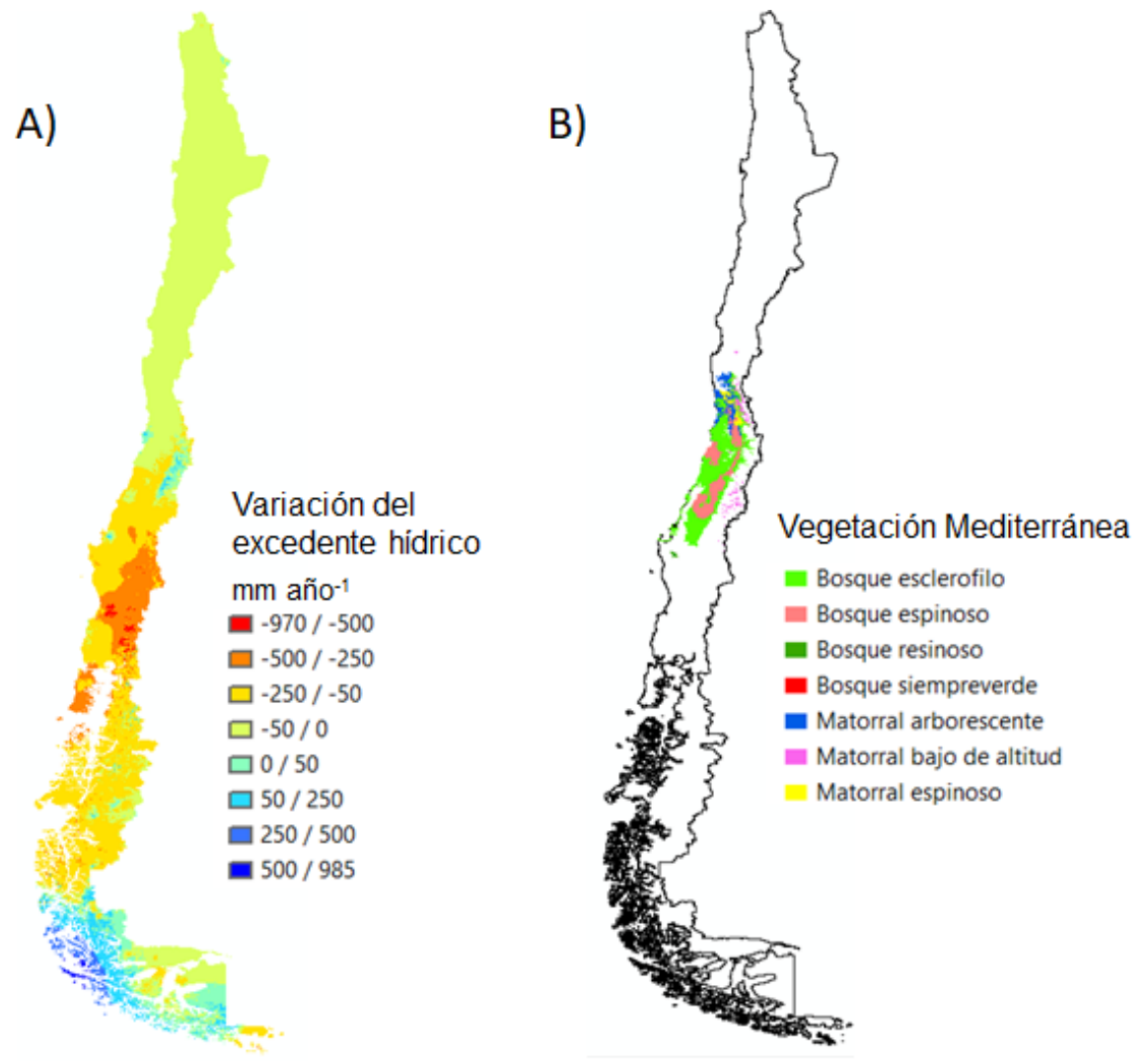

Figura 5: Variación de los recursos hídricos en Chile para el año 2050, A); y distribución actual de la vegetación mediterránea, B). Los valores del mapa A resultan de la diferencia entre el escenario RCP4.5 del año 2050 y el periodo base (1970-2000).

Los espacios con vegetación mediterránea podrían soportar unos descensos de sus recursos de hasta $250 \mathrm{~mm}$ año-1 (Figura 5.A y B), con zonas puntuales donde las reducciones podrían ser de hasta $340 \mathrm{~mm}_{\text {año-1 }}$ (Tabla 1). Las formaciones vegetales con mayor representación superficial de la zona mediterránea chilena, el bosque esclerófilo y el bosque espinoso, podrían reducir sus recursos hídricos en un promedio de aproximadamente $75 \mathrm{~mm}$ año-1 respecto al periodo base (Tabla 1). Esto significa 
que el $80 \%$ de este espacio deberá hacer frente a unas características ambientales mucho más secas que las existentes en la actualidad.

Tabla 1: Variación de los recursos hídricos en Chile para el año 2050 en las distintas formaciones vegetales mediterráneas. Los valores resultan de la diferencia entre el escenario RCP4.5 del año 2050 y el periodo base (1970-2000). DE = Desviación estándar.

\begin{tabular}{|c|c|c|c|c|c|}
\hline Formación vegetal & $\begin{array}{c}\text { Superficie } \\
(\%)\end{array}$ & $\begin{array}{c}\text { Media } \\
\left(\mathrm{mm} \mathrm{año-1}^{-1}\right)\end{array}$ & $\begin{array}{c}\mathrm{DE} \\
\left(\mathrm{mm} \mathrm{año}^{-1}\right)\end{array}$ & $\begin{array}{c}\text { Mínimo } \\
\left(\mathrm{mm} \mathrm{año}^{-1}\right)\end{array}$ & $\begin{array}{c}\text { Máximo } \\
\left(\mathrm{mm}^{\text {año }}{ }^{-1}\right)\end{array}$ \\
\hline Bosque esclerófilo & 55.63 & -73.6 & 73.8 & -340.0 & 157.2 \\
\hline Bosque espinoso & 25.06 & -75.9 & 54.2 & -201.1 & 21.3 \\
\hline Bosque resinoso & 0.44 & -120.6 & 12.3 & -158.2 & -96.0 \\
\hline Bosque perenne & 0.03 & -275.7 & 13.5 & -296.2 & -234.3 \\
\hline Matorral arborescente & 10.23 & -5.1 & 10.1 & -59.4 & 14.3 \\
\hline Matorral bajo de altitud & 5.04 & -44.3 & 55.8 & -326.6 & 59.5 \\
\hline Matorral espinoso & 3.56 & -9.3 & 9.9 & -44.5 & 0.0 \\
\hline
\end{tabular}

Por otro lado, el descenso de los recursos en la zona central tiene la particularidad de ocurrir entre las ciudades de Santiago y Valparaíso, las más pobladas. Esto resulta en una reducción significativa de los recursos hídricos en la zona central de Chile, donde hay mayor concentración de población y donde el clima mediterráneo, caracterizado por su impredecible variabilidad, es predominante. Estudios como el desarrollado por Lozano-Parra et al. (2018a; b), señalan que el agua es el principal elemento que controla la dinámica de biomasa en zonas de clima mediterráneo y que cuando las condiciones hídricas se vuelven secas, la producción de biomasa puede descender hasta un $50 \%$, comprometiendo sus funciones ambientales y económicas. Asimismo, cuando se producen déficits de humedad de suelo prolongados y elevados, los flujos de calor sensible dominan sobre los flujos de calor latente, pudiendo dar lugar a una atmósfera más cálida que inhibe la formación de nubes por convección, creando un circuito de retroalimentación positivo (Lozano-Parra et al., 2018c; Scheffer et al., 2001). Este hecho podría afectar directamente sobre episodios de calor extremo a escala local, tal y como ya lo describió Alexander (2011) para regiones relativamente secas del sudeste de Europa. Por ello, se hace necesario continuar analizando las interacciones entre procesos ecohidrológicos y variables meteorológicas, con el fin de evaluar el posible impacto de los déficits hídricos sobre las condiciones climáticas locales en los ecosistemas mediterráneos (Lewis et al., 2000; Seneviratne et al., 2010).

\section{Conclusiones}

Chile es un país rico en recursos hídricos. Sin embargo, su distribución espaciotemporal y sus variaciones futuras dan lugar a una disponibilidad muy desequilibrada. El estudio de los recursos hídricos adquiere mayor relevancia al considerar que la zona central constituye uno de los espacios con menos excedentes y donde se prevé uno de los mayores descensos de sus recursos hídricos. Asimismo, a pesar de que la vegetación de esta zona soporta una gran variedad de actividades económicas y 
ambientales, como los viñedos o los sistemas extensivos, está expuesta a una reducción de los recursos y la impredecibilidad intrínseca del clima mediterráneo. Descifrar los mecanismos y procesos ecohidrológicos que controlan a los ecosistemas y determinar su sensibilidad ante las perturbaciones ambientales constituye uno de los desafíos más importantes de la ecohidrología, a fin de entender los espacios y gestionar sus recursos.

\section{Agradecimentos}

Los autores agradecen al gobierno de Chile por la financiación proporcionada mediante los proyectos FONDECYT 11161097 y REDI170640, que han posibilitado desarrollar este estudio.

\section{Bibliografia}

Asbjornsen, H., Goldsmith, G. R., Alvarado-Barrientos, M. S., Rebel, K., Osch, F. P. V., Rietkerk, M., et al. (2011). Ecohydrological advances and applications in plant-water relations research: a review. Journal of Plant Ecology, 4, 3-22. https://doi.org/10.1093/jpe/rtr005

Blöschl, G., Bierkens, M. F. P., Chambel, A., Cudennec, C., Destouni, G., Fiori, A., et al. (2019). Twenty-three unsolved problems in hydrology (UPH) - a community perspective. Hydrological Sciences Journal. https://doi.org/10.1080/02626667.2019.1620507

Eagleson, P. (2002). Ecohydrology: Darwinian expression of vegetation form and function. UK: Cambrige University Press.

FAO (2014). World reference base for soil resources 2014. International soil classification system for naming soils and creating legends for soil maps (Vol. 106). Rome: FAO. 106.

Fick, S., Hijmans, R. (2017). WorldClim 2: new 1-km spatial resolution climate surfaces for global land areas. International Journal of Climatology, 37, 4302-4315. https://doi.org/10.1002/joc.5086

García Marín, R., Schnabel, S., Pulido Fernández, M., Lozano-Parra, F. J., Jariego García, Á., Lagar Timón, D. (2010). Riesgo de sequía y gestión de recursos hídricos. In J. Mora Aliseda, F. Dos Reis Condesso, \& B. De Sao Pedro (Eds.), Gestión sostenible de los recursos hídricos (pp. 445-473). Lisboa, Portugal.

Hearne, R., Donoso, G. (2014). Water markets in Chile: Are they meeting needs? In W. Easter \& Q. Huang (Eds.), Water markets for the 21st Century. What have we learned? Global Issues in Water Policy (Vol. 11, pp. 103-126). USA: Springer.

IPCC (2014). Climate Change 2014: Synthesis Report. Contribution of Working Groups I, II and III to the Fifth Assessment Report of the Intergovernmental Panel on Climate Change. Geneva, Switzerland.

Larraín, S., Poo, P. (2010). Conflictos por el Agua en Chile. Entre los derechos humanos y las reglas del mercado. Chile: Embajada de Holanda y Fundación Heinrich Böll.

Lewis, D., Singer, M. J., Dahlgren, R. A., Tate, K. W. (2000). Hydrology in a California oak woodland watershed: a 17-year study. Journal of Hydrology, 240, 106-117. https://doi.org/10.1016/S0022-1694(00)00337-1 
Lozano-Parra, J. (2015). Dinámica del agua edáfica en dehesas y su relación con el clima y la vegetación. Boletín de la Asociación de Geográfos Españoles, 69, 625-629.

Lozano-Parra, J., Maneta, M., Schnabel, S. (2014). Climate and topographic controls on simulated pasture production in a semiarid Mediterranean watershed with scattered tree cover. Hydrology and Earth System Sciences, 18, 1439-1456. https://doi.org/10.5194/hess18-1439-2014

Lozano-Parra, J., Schnabel, S. (2015). Respuesta de la vegetación herbácea a las variaciones hídricas del suelo. In S. Martínez Pérez \& A. Sastre Merlín (Eds.), Estudios de la Zona No Saturada (Vol. XII, pp. 77-84). Alcalá de Henares: Universidad de Alcalá de Henares.

Lozano-Parra, J., Schnabel, S., Ceballos-Barbancho, A. (2015). The role of vegetation covers on soil wetting processes at rainfall event scale in scattered tree woodland of Mediterranean climate. Journal of Hydrology, 529, 951-961. https://doi.org/10.1016/j.jhydrol.2015.09.018

Lozano-Parra, J., Van Schaik, L., Schnabel, S., Gómez-Gutiérrez, Á. (2016). Soil moisture dynamics at high temporal resolution in a mediterranean watershed with scattered tree cover. Hydrological Processes, 30, 1155-1170. https://doi.org/10.1002/hyp.10694

Maneta, M. P., Soulsby, C., Kuppel, S., Tetzlaff, D. (2018). Conceptualizing catchment storage dynamics and nonlinearities. Hydrological Processes, Invited Commentary, 1-5. https://doi.org/10.1002/hyp.13262

McColl, K., Alemohammad, S., Akbar, R., Konings, A., Yueh, S., Entekhabi, D. (2017). The global distribution and dynamics of surface soil moisture. Nature Geoscience. https://doi.org/10.1038/ngeo2868

Pliscoff, P., Luebert, F. (2006). Sinopsis Bioclimatica y Vegetacional de Chile. Editorial Universitaria.

Rivera, D., Godoy-Faúndez, A., Lillo, M., Alvez, A., Delgado, V., Gonzalo-Martín, C., et al. (2016). Legal disputes as a proxy for regional conflicts over water rights in Chile. Journal of Hydrology, 535, 36-45. https://doi.org/10.1016/j.jhydrol.2016.01.057

Rodell, M., Famiglietti, J., Wiese, D., Reager, J., Beaudoing, H., Landerer, F., et al. (2018). Emerging trends in global freshwater availability. Nature, 557, 651-659. https://doi.org/10.1038/s41586-018-0123-1

Rodríguez-Iturbe, I. (2000). Ecohydrology: A hydrologic perspective of climate-soil-vegetation dynamics. Water Resources Research, 36, 3-9. https://doi.org/10.1029/1999WR900210

Rodríguez-Iturbe, I., D'Odorico, P., Laio, F., Ridolfi, L., Tamea, S. (2007). Challenges in humid land ecohydrology: Interactions of water table and unsaturated zone with climate, soil, and vegetation. Water Resources Research, 43, 1-5. https://doi.org/10.1029/2007WR006073

Rodríguez-Iturbe, I., Porporato, A. (2004). Ecohydrology of Water-Controlled Ecosystems. Cambrige, UK: Cambrige University Press.

Rubel, F., Kottek, M. (2010). Observed and projected climate shifts 1901-2100 depicted by world maps of the Koppen-Geiger climate classification. Meteorologische Zeitschrift, 19, 135-141. https://doi.org/10.1127/0941-2948/2010/0430

Scheffer, M., Carpenter, S., Foley, J. A., Folke, C., Walker, B. (2001). Catastrophic shifts in ecosystems. Nature, 413, 591-596. https://doi.org/10.1038/35098000 
Scheffer, M., Carpenter, S. R. (2003). Catastrophic regime shifts in ecosystems: linking theory to observation. Trends in Ecology and Evolution, 18, 648-656. https://doi.org/10.1016/j.tree.2003.09.002

Seneviratne, S., Corti, T., Davin, E., Hirschi, M., Jaeger, E., Lehner, I., et al. (2010). Investigating soil moisture-climate interactions in a changing climate: A review. EarthScience Reviews, 99, 125-161. https://doi.org/10.1016/j.earscirev.2010.02.004

Turc, L. (1961). Estimation of irrigation water requirements, potential evapotranspiration: A simple climatic formula evolved up to date. Ann. Agron., 12, 13-49.

Wahba, G. (1990). Spline Models for Observational Data. Paper presented at the CBMS-NSF Regional Conference Series in Applied Mathematics, University of Wisconsin, Madison, Wisconsin.

World Bank. (2011). Chile: Diagnóstico de la gestión de los recursos hídricos. In W. Bank (Ed.), Banco Mundial (Vol. N 63392, pp. 88).

Artigo recebido em/ Received on: 03/09/2019

Artigo aceite para publicação em/ Accepted for publication on: 24/04/2020 